\title{
Passive control of global instability in low-density jets
}

\author{
Ubaid Ali Qadri*, Gary J. Chandler, Matthew P. Juniper \\ Department of Engineering, University of Cambridge, Trumpington Street, Cambridge, CB2 \\ $1 P Z, U K$
}

\begin{abstract}
Many studies have shown that low-density jets exhibit self-excited varicose oscillations. We use direct numerical simulation of the low Mach number NavierStokes equations to perform a linear global stability analysis of a helium jet at the threshold of onset of these oscillations. We calculate the direct and adjoint global modes and overlap these to obtain the structural sensitivity. We find that the structural sensitivity has high magnitudes in the shear layer downstream of the entrance plane, where the flow is absolutely unstable. We use the direct and adjoint global modes to calculate the effect of a control force on the growth rate and frequency of the unstable mode. We produce maps of the regions of the flow that are most sensitive to localized open loop steady forcing in the form of a body force and a heat source. We find that the most sensitive location for open loop steady forcing is the area around the shear layer, around 2 jet diameters downstream of the exit plane, and that the influence of steady forcing and heat injection is advected by the flow outside the jet. We use these maps to calculate the influence of a ring placed in the flow. When the ring is at the same temperature as the flow, it influences the flow through its drag. The ring has most influence when placed in the inner edge of the shear layer. When the ring is heated, it also influences the flow through the density reduction caused by heat input. In this case, the ring has most influence when placed in the outer edge of the shear layer. It is also influential when placed outside the jet because the expanded gas is advected towards the jet. In both these cases, the influence
\end{abstract}

\footnotetext{
* Corresponding author
} 
of the steady change to the base flow is significantly greater than the influence of an unsteady feedback force caused by the ring.

Keywords: instability, flow control, jets and wakes

\section{Introduction}

Many studies have shown that the stability of a jet discharging into an ambient fluid depends strongly on the jet's density. For example, spectra of hot-wire velocity measurements in helium-air jets [1] and pressure measurements in heated air jets [2] showed that low-density jets exhibit sharp discrete peaks in the measured spectra, while uniform-density jets exhibit weak broad peaks. These sharp discrete peaks are caused by self-excited varicose oscillations in the low-density jets, which arise because the initially steady jet is globally unstable. This global instability arises from a region of local absolute instability at the jet exit plane. $[3,4,5,6]$ Theoretical studies have shown that global instability arises due to a hydrodynamic feedback mechanism in the region of local absolute instability. $[7,8]$

Several experimental studies have examined the control of these self-excited oscillations. These studies have used loudspeakers for active feedback control [9], and thin hot-wires [10] or co-flow [11] for passive control. The goal of such control is to render the jet globally stable by perturbing the base flow and disrupting the feedback mechanism that drives the oscillations. For this goal, a global linear stability analysis around the steady base flow is the most appropriate tool, particularly in the form of a base flow sensitivity analysis $[12,13]$. These analyses require the direct global mode and the adjoint global mode to be calculated.

In this numerical study, we perform a base flow sensitivity analysis on a low-density jet. We use the low Mach number formulation of the Navier-Stokes (NS) equations [14]. This is well-adapted to studies of hydrodynamic instability in low-density jets and flames because it permits density variations due to temperature and composition but excludes acoustic waves. This significantly 
reduces the computational effort. We use the direct and adjoint global modes to identify the effect of regions of the flow in which the introduction of a thin axisymmetric control ring can change the frequency or growth rate of the global instability. We also determine the influence of heat transfer from the ring, which has a particularly strong influence on the instability of a low-density jet.

\section{Flow configuration}

We study the axisymmetric motion of a low-density jet in a cylindrical domain that has radius $R_{\max }$ and length $X_{\max }$. The jet fluid enters the domain at $x=0$ and is aligned along the axis, $r=0$. The fluid in the domain is described in terms of its velocity $\mathbf{u}=\left(u_{x}, u_{r}\right)^{T}$, density $\rho$, and temperature $T$. A difference in chemical species between the jet fluid and the surrounding fluid is described using the mixture fraction, $Z$, which has a value of $Z=1$ for the jet species and $Z=0$ for the surrounding fluid.

The fluid motion is described by the axisymmetric low Mach number (LMN) equations in nondimensional form:

$$
\begin{aligned}
\frac{\partial \rho}{\partial t}+\nabla \cdot(\rho \mathbf{u}) & =0 \\
\frac{\partial(\rho \mathbf{u})}{\partial t} & =-\nabla p+\nabla \cdot\left(\frac{1}{S_{1} R e} \tau-\rho \mathbf{u u}\right)+\operatorname{Ri}(1-\rho) \hat{\mathbf{g}} \\
\rho\left(\frac{\partial Z}{\partial t}+\mathbf{u} \cdot \nabla Z\right) & =\frac{1}{S_{1} \operatorname{ReSc}} \nabla^{2} Z \\
\rho\left(\frac{\partial T}{\partial t}+\mathbf{u} \cdot \nabla T\right) & =\frac{1}{S_{1} \operatorname{RePr}} \nabla^{2} T \\
\rho\left[\left(S_{1}-1\right) Z+1\right]\left[\left(S_{2}-1\right) T+1\right] & =1
\end{aligned}
$$

where $\tau=\left[\nabla \mathbf{u}+(\nabla \mathbf{u})^{T}\right]-\frac{2}{3}(\nabla \cdot \mathbf{u}) I$ is the non-isotropic component of the rate-of-strain tensor. The flow variables are nondimensionalized by the jet diameter, the jet axial velocity at inlet, and the ambient density. The Reynolds number, Re, is defined in terms of the jet diameter, the jet axial velocity at inlet, and the jet density. This definition introduces a $1 / S_{1}$ factor in front of 
the viscous terms in equations (1). The nondimensional temperature is defined as $T=\left(T^{*}-T_{0}\right) /\left(T_{1}-T_{0}\right)$, where $T^{*}$ is the dimensional temperature, $T_{1}$ is the maximum temperature, and $T_{0}$ is the ambient temperature. The ratio of the ambient density to the jet density defines the density ratio parameter, $S_{1}$, and the ratio of the maximum temperature to the ambient temperature defines the temperature ratio parameter, $S_{2}$. The Prandtl number, Pr, and Schmidt number, Sc, describe the ratio of the diffusivity of temperature and mass, respectively, to the diffusivity of momentum. In this study, we wish to model an isothermal helium jet exiting into atmospheric conditions. This flow has a density ratio of $S_{1}=7.0$. For an isothermal flow, $S_{2}$ can be set to any value other than 1.0. This is because, for an isothermal flow, it defines a nominal temperature to nondimensionalize the equations of motion. We set $S_{2}=2.0$ for simplicity. In this fundamental study, we assume that the viscosity and thermal diffusivity are uniform throughout the flow, and set $\operatorname{Pr}=S c=1.0$, in line with Lesshaft et al [3]. The Richardson number, Ri, represents the ratio of the bouyancy force to the inertial force. In this study, we are interested in the case where buoyancy effects are negligible and the dynamics of the low-density jet are dominated by the inertial force, and therefore, we set $R i=0$. Such a flow configuration is typical of previous experimental studies [15, 11, 9, 16]. Global instability has also been observed in buoyant jets [17], but is not examined in this study.

Equations (1a)-(1e) can be expressed in terms of the momentum, $\mathbf{m} \equiv \rho \mathbf{u}$, temperature, $T$, and mixture fraction, $Z$, as

$$
\frac{\partial \mathbf{q}}{\partial t}=\mathcal{N}(\mathbf{q})
$$

where $\mathbf{q} \equiv\left(m_{x}, m_{r}, Z, T\right)^{T}$ is the state vector and $\mathcal{N}(\cdot)$ is a nonlinear differential operator representing the action of the equations on the state vector. The density, $\rho$, is not included in the state vector because it can be derived from $T$ and $Z$. We use a direct numerical simulation (DNS) code from previous studies $[18,19]$ to solve these equations. A fourth-order Runge-Kutta scheme is used to march the discretized equations forward in time. The equations are discretized 
in space using a sixth-order compact finite difference scheme. We use a grid with $255 \times 2049$ points for a domain measuring $8.0 \times 36.0$ jet diameters in the radial and axial directions respectively. We assess numerical convergence in Appendix A, where we compare the growth rates and frequencies of the global modes produced on a range of computational domains and mesh resolutions.

Along the lateral boundary, at $r=R_{\max }$, we use a viscous traction free boundary condition for the momentum and set $T=0$ and $Z=0$. At the outlet boundary, at $x=X_{\max }$, we use a convective boundary condition for the momentum, temperature and mixture fraction. These boundary conditions model flow into a semi-infinite domain in the downstream and radial directions. The pressure-projection scheme used in the code uses a discrete cosine transform to set boundary conditions for the pressure at the inlet and outlet boundaries. For this study, we use a half-wave cosine transform, which sets $d p / d x=0$ at the inlet and outlet boundaries. Along the lateral boundary, we set $p=0$. At the inlet to the domain, we impose velocity and mixture fraction profiles formed from Michalke's profile number two [20], with a shear-layer thickness parameter $D / 2 \theta_{0}=14.0$. This signifies that the momentum thickness of the shear layer is 14 times smaller than the jet radius. We also add a co-flow velocity of $1 \%$ of the jet velocity around the jet to improve numerical stability.

This study is performed near the threshold of global instability, at which point a linear global stability analysis is most relevant to the fully nonlinear case. For this set of parameters and inlet profile, we find that this is at $R e=470$. The linear global mode has a frequency of $S t=0.165$. In comparison, Hallberg \& Strykowski [15] (Figure 5) report the onset of global instability in a helium jet of similar shear-layer thickness at approximately $550 \leq \mathrm{Re} \leq 750$ and find that the nonlinear global mode has a frequency of $0.18 \leq S t \leq 0.22$. We attribute the difference between our linear global stability analysis and their experimental observations to the differences in the jet velocity and density profiles. Both of these have been found to have a significant effect on the onset of absolute instability in low-density jets. [5, 21] 


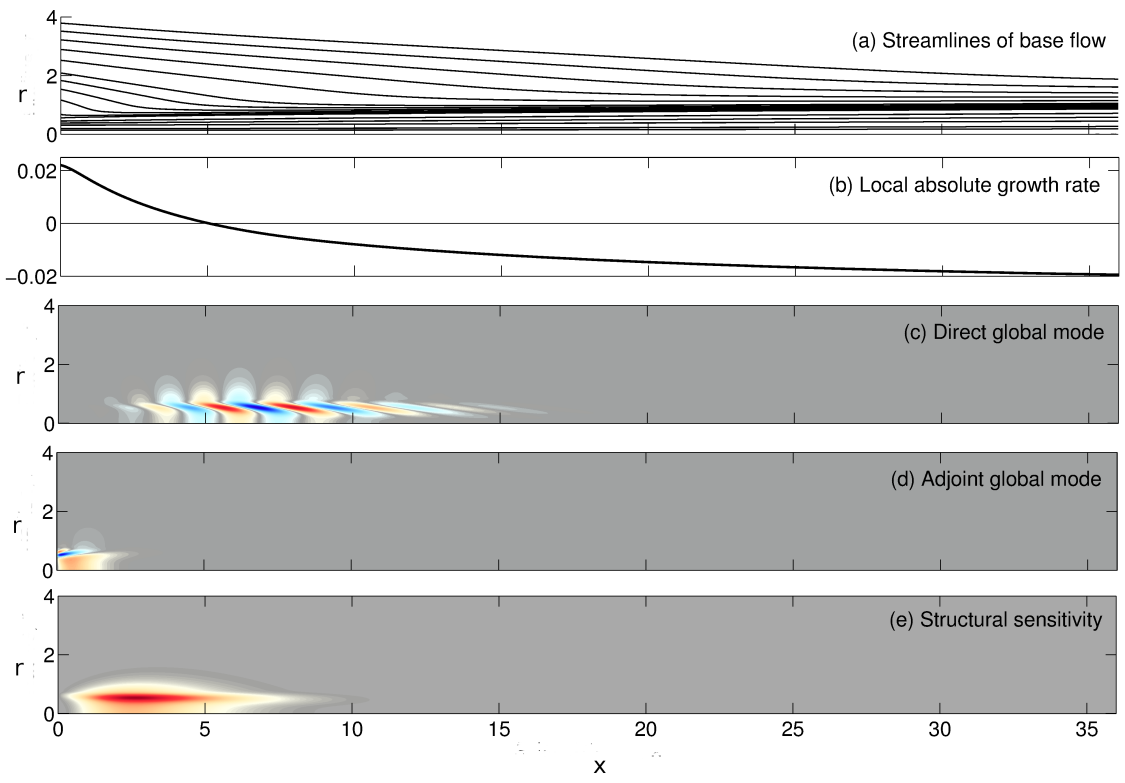

Figure 1: (a) Streamlines of the base flow and (b) the local absolute growth rate of an isothermal helium jet at $\operatorname{Re}=470$. (c) The direct global mode and (d) the adjoint global mode, shown as the real part of the axial momentum. (e) The structural sensitivity, with a maximum of $4 \times 10^{3}$.

\section{Global stability analysis}

We obtain a steady axisymmetric base flow, $\overline{\mathbf{q}}(x, r)$, such that $\mathcal{N}(\overline{\mathbf{q}})=0$, using selective frequency damping (SFD) [22]. The streamlines of this base flow are shown in figure 1(a). The entrainment of the ambient fluid into the jet is significant and we will show later in the paper that it can have a strong effect on the stability of the flow.

The evolution of small axisymmetric perturbations $\mathbf{q}^{\prime}$ around this steady base flow is governed by the linearized LMN equations. We decompose the axisymmetric perturbations into Fourier modes in time:

$$
\mathbf{q}^{\prime}(x, r, t)=\hat{\mathbf{q}}(x, r) e^{\lambda t}+\text { complex conjugate },
$$

where $\lambda \equiv \sigma+\mathrm{i} \omega$ is the eigenvalue. This contains the growth rate, $\sigma$, and frequency, $\omega$, of the corresponding two-dimensional eigenmode, $\hat{\mathbf{q}}(x, r)$, that 
would grow or decay on top of the steady base flow. The direct global modes are obtained by solving the matrix eigenvalue problem

$$
\lambda \hat{\mathbf{q}}=\mathbf{L} \hat{\mathbf{q}},
$$

where $\mathbf{L}$ is a discretized operator that describes the linearized LMN equations. We also obtain the adjoint global modes, which are solutions of

$$
\lambda^{*} \hat{\mathbf{q}}^{+}=\mathbf{L}^{+} \hat{\mathbf{q}}^{+}
$$

where $\mathbf{L}^{+}$is the discretized version of the continuous-adjoint LMN equations. We solve these eigenvalue problems using the code developed by Chandler et al [19]. The code uses matrix-free time-stepping and the implicitly restarted Arnoldi method to find the most unstable global modes. For the direct global modes, we use a convective boundary condition at the outlet boundary and set $\hat{m}_{x}=\hat{m}_{r}=\hat{T}=\hat{Z}=0$ on the lateral and inlet boundaries. For the adjoint global modes, we set $\hat{m}_{x}^{+}=\hat{m}_{r}^{+}=\hat{T}^{+}=\hat{Z}^{+}=0$ on all boundaries. The halfwave cosine transform used in the code sets $d \hat{p} / d x=d \hat{p}^{+} / d x=0$ at the inlet and outlet boundaries. We find that this gives better agreement between the direct and continuous-adjoint eigenvalues than that observed by Chandler et al [19], who used a quarter-wave cosine transform to set $d \hat{p} / d x=\hat{p}^{+}=0$ at the inlet and $\hat{p}=d \hat{p}^{+} / d x=0$ at the outlet.

Figure 2 shows the eigenvalues corresponding to the 25 least stable modes. At this Reynolds number, we find one mode that is marginally unstable, and a branch of low-frequency stable modes. These stable modes correspond to free-stream vortical modes - similar to those observed in a uniform-density jet [23].

The direct global mode, $\hat{\mathbf{q}}(x, r)$, corresponding to the marginally unstable mode is shown in figure 1(c). Its corresponding adjoint global mode, $\hat{\mathbf{q}}^{+}(x, r)$, is shown in figure 1(d) and the structural sensitivity, defined here as the Frobenius norm of the tensor $S_{i j}=\hat{\mathbf{m}}_{i}\left(\hat{\mathbf{m}}_{j}^{+}\right)^{*}$, is in figure 1(e).

The absolute growth rate decays monotonically from the entrance plane and the flow is absolutely unstable for $0 \leq x<5.0$. The structural sensitivity has 


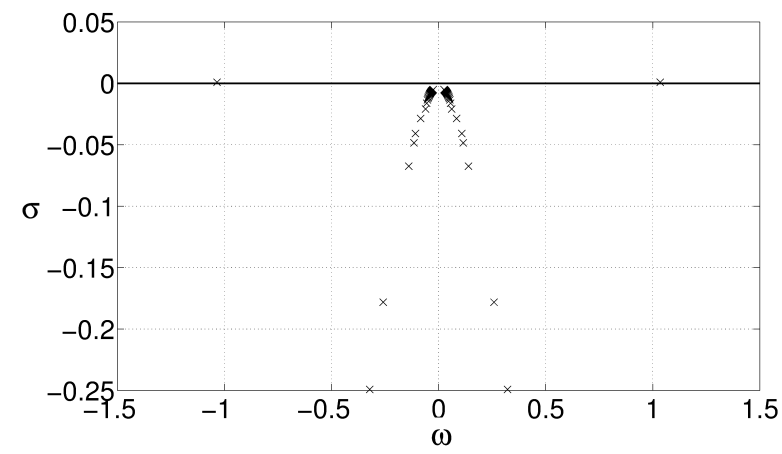

Figure 2: Eigenvalue spectrum for axisymmetric modes for the base flow shown in figure 1(a). The 25 least stable modes are shown.

high magnitudes in the shear layer in this region. The structural sensitivity identifies the region of the flow that is most sensitive to internal feedback mechanisms - where the direct global mode optimally excites itself. We follow earlier studies [24] and refer to this region as the wavemaker of the flow. This region does not, however, necessarily correspond to the region where an external control force has most influence on the growth rate and frequency of the unstable mode. This is because a control force also changes the base flow and this change in the base flow is not accounted for in the structural sensitivity.

\section{Sensitivity to a control force}

We now consider the effect of a small control force on the marginally unstable eigenvalue. We model the control force by adding source terms to the right-hand side of equation (2):

$$
\frac{\partial \mathbf{q}}{\partial t}=\mathcal{N}(\mathbf{q})+\mathbf{F}
$$

where $\mathbf{F} \equiv\left(\varrho, \mathbf{f}, 0, \psi_{T}\right)^{T}$ contains the source terms added to the right-hand side of the continuity, momentum, species and energy equations respectively. The source term in the species equation has been set to zero. The variables $\varrho$, f and $\psi_{T}$ are the non-dimensional rates of addition per unit volume of mass, 
momentum, and thermal energy into the flow. In this linear stability framework, the control force has a steady component, $\overline{\mathbf{F}}$, that acts on the base flow, $\overline{\mathbf{q}}$, and a linearized perturbation, $\mathbf{f}^{\prime}$, that acts on the linear perturbations, $\mathbf{q}^{\prime}$. The effects of these two components are modelled separately following the approach of Marquet et al [25].

The eigenvalue of the global mode, $\lambda$, is a function of the base flow fields, $\overline{\mathbf{q}}$, and these are, in turn, functions of the steady components of the forcing terms, $\overline{\mathbf{F}}$. The effect of $\overline{\mathbf{F}}$ on $\lambda$ is calculated by formulating a constrained Lagrangian problem

$$
\mathcal{L}=\lambda-\left\langle\overline{\mathbf{q}}^{+}, \mathcal{N}(\overline{\mathbf{q}})+\overline{\mathbf{F}}\right\rangle-\left\langle\hat{\mathbf{q}}^{+}, \lambda \hat{\mathbf{q}}-\mathbf{L} \hat{\mathbf{q}}\right\rangle
$$

and calculating $\nabla_{\overline{\mathbf{F}}} \lambda$, the functional derivative of $\lambda$ with respect to (w.r.t) $\overline{\mathbf{F}}$. This is labelled the sensitivity of the eigenvalue to steady forcing. The nonlinear and linearized Navier-Stokes equations act as constraints in this problem. The notation $\langle\mathbf{a}, \mathbf{b}\rangle$ denotes an inner product over the computational domain volume $V$,

$$
\langle\mathbf{a}, \mathbf{b}\rangle=\frac{1}{V} \int_{V} \mathbf{a}^{H} \mathbf{b} d V
$$

where $\mathbf{a}^{H}$ denotes the Hermitian (i.e. complex conjugate transpose) of $\mathbf{a}$. The Lagrange multipliers, $\overline{\mathbf{q}}^{+}$and $\hat{\mathbf{q}}^{+}$, are the adjoint base flow and adjoint global mode fields respectively.

The sensitivity to steady forcing is equal to the functional derivative of $\mathcal{L}$ w.r.t $\overline{\mathbf{F}}$ when all the constraints are satisfied. To find this, we first set the functional derivatives of $\mathcal{L}$ w.r.t all other variables to zero. The derivative w.r.t $\hat{\mathbf{q}}$ leads to a set of equations that defines the eigenvalue problem for the adjoint global mode, $-\lambda^{*} \hat{\mathbf{q}}^{+}+\mathbf{L}^{+} \hat{\mathbf{q}}^{+}=0$. The derivative w.r.t $\lambda$ leads to the normalization condition $\left\langle\hat{\mathbf{m}}^{+}, \hat{\mathbf{m}}\right\rangle+\left\langle\hat{Z}^{+}, \hat{Z}\right\rangle+\left\langle\hat{T}^{+}, \hat{T}\right\rangle=1$. The derivative w.r.t $\overline{\mathbf{q}}$ leads to a set of equations for the adjoint base flow fields, $\overline{\mathbf{L}}^{+} \overline{\mathbf{q}}^{+}=\overline{\mathbf{g}}^{+}$. The derivative w.r.t $\overline{\mathbf{F}}$ then shows that the sensitivity of the eigenvalue to steady forcing is obtained from the relevant adjoint base flow field. Similarly, the sensitivity of the eigenvalue to unsteady harmonic forcing is obtained from the relevant adjoint global mode field. 
For incompressible flow, the adjoint base flow operator $\overline{\mathbf{L}}^{+}$is equivalent to the continuous-adjoint operator $\mathbf{L}^{+}$. [12] For our formulation of the LMN equations, however, these two operators are different. The adjoint base flow equations can be written in full as

$$
\begin{gathered}
\frac{\partial \bar{m}_{i}^{+}}{\partial x_{i}}=0 \\
-\frac{\bar{m}_{j}}{\bar{\rho}}\left(\frac{\partial \bar{m}_{i}^{+}}{\partial x_{j}}-\frac{\partial \bar{m}_{j}^{+}}{\partial x_{i}}\right)-\frac{\partial \bar{p}^{+}}{\partial x_{i}}-\frac{1}{S_{1} \operatorname{Re} \bar{\rho}}\left(\frac{\partial^{2} \bar{m}_{i}^{+}}{\partial x_{j}^{2}}+\frac{1}{3} \frac{\partial^{2} \bar{m}_{j}^{+}}{\partial x_{j} \partial x_{i}}\right)+\bar{T}^{+} \frac{\partial \bar{T}}{\partial x_{i}}+\bar{Z}^{+} \frac{\partial \bar{Z}}{\partial x_{i}}=\bar{f}_{i}^{+}
\end{gathered}
$$

$$
\begin{aligned}
& -\bar{m}_{i} \frac{\partial \bar{Z}^{+}}{\partial x_{i}}-\frac{1}{S_{1} \operatorname{ReSc}} \frac{\partial^{2} \bar{Z}^{+}}{\partial x_{i}^{2}}+\bar{K}_{2} \bar{\rho} \bar{\rho}^{+}=\bar{\psi}_{Z} \\
& -\bar{m}_{i} \frac{\partial \bar{T}^{+}}{\partial x_{i}}-\frac{1}{S_{1} \operatorname{RePr} r} \frac{\partial^{2} \bar{T}^{+}}{\partial x_{i}^{2}}+\bar{K}_{1} \bar{\rho} \bar{\rho}^{+}=\bar{\psi}_{T} \\
& \frac{\bar{m}_{i} \bar{m}_{j}}{\bar{\rho}^{2}} \frac{\partial \bar{m}_{i}^{+}}{\partial x_{j}}+\frac{\bar{m}_{i}}{S_{1} R e \bar{\rho}^{2}}\left(\frac{\partial^{2} \bar{m}_{i}^{+}}{\partial x_{j}^{2}}+\frac{1}{3} \frac{\partial^{2} \bar{m}_{j}^{+}}{\partial x_{j} \partial x_{i}}\right)+\frac{\bar{\rho}^{+}}{\bar{\rho}}=\bar{\varrho}^{+}
\end{aligned}
$$

where $\bar{K}_{1} \equiv\left(S_{2}-1\right)\left(\left(S_{1}-1\right) \bar{Z}+1\right)$ and $\bar{K}_{2} \equiv\left(S_{1}-1\right)\left(\left(S_{2}-1\right) \bar{T}+1\right)$ are constant scalar fields. The complex fields that constitute $\overline{\mathbf{g}}^{+} \equiv\left(0, \bar{f}_{i}^{+}, \bar{\psi}_{Z}^{+}\right.$, $\left.\bar{\psi}_{T}^{+}\right)^{T}$ and $\bar{\varrho}^{+}$on the RHS need to be calculated first from the base flow and the 
direct and adjoint global modes as

$$
\begin{aligned}
& \bar{f}_{i}^{+} \equiv\left(\frac{\hat{m}_{j}^{*}}{\bar{\rho}}-\frac{\hat{\rho}^{*} \bar{m}_{j}}{\bar{\rho}^{2}}\right)\left(\frac{\partial \hat{m}_{i}^{+}}{\partial x_{j}}+\frac{\partial \hat{m}_{j}^{+}}{\partial x_{i}}\right)-\frac{\hat{\rho}^{*}}{S_{1} \operatorname{Re} \bar{\rho}^{2}}\left(\frac{\partial^{2} \hat{m}_{i}^{+}}{\partial x_{j}^{2}}+\frac{1}{3} \frac{\partial^{2} \hat{m}_{j}^{+}}{\partial x_{j} \partial x_{i}}\right) \cdots \\
& \cdots-\left(\frac{\hat{T}^{+}}{\bar{\rho}}+\bar{K}_{1} \hat{p}^{+}\right) \frac{\partial \hat{T}^{*}}{\partial x_{i}}-\left(\frac{\hat{Z}^{+}}{\bar{\rho}}+\bar{K}_{2} \hat{p}^{+}\right) \frac{\partial \hat{Z}^{*}}{\partial x_{i}}, \\
& \bar{\psi}_{Z}^{+} \equiv \frac{\partial}{\partial x_{j}}\left(\frac{\hat{Z}^{+} \hat{m}_{j}^{*}}{\bar{\rho}}\right)-\left(S_{2}-1\right)\left(S_{1}-1\right) \hat{p}^{+}\left(\bar{m}_{j} \frac{\partial \hat{T}^{*}}{\partial x_{j}}-\frac{1}{S_{1} \operatorname{RePr}} \frac{\partial^{2} \hat{T}^{*}}{\partial x_{j}^{2}}\right) \cdots \\
& \cdots-\left(S_{2}-1\right)\left(S_{1}-1\right) \hat{\rho}^{+} \hat{T}^{*}, \\
& \bar{\psi}_{T}^{+} \equiv \frac{\partial}{\partial x_{j}}\left(\frac{\hat{T}^{+} \hat{m}_{j}^{*}}{\bar{\rho}}\right)-\left(S_{2}-1\right)\left(S_{1}-1\right) \hat{p}^{+}\left(\bar{m}_{j} \frac{\partial \hat{Z}^{*}}{\partial x_{j}}-\frac{1}{S_{1} \operatorname{ReSc}} \frac{\partial^{2} \hat{Z}^{*}}{\partial x_{j}^{2}}\right) \cdots \\
& \cdots-\left(S_{2}-1\right)\left(S_{1}-1\right) \hat{\rho}^{+} \hat{Z}^{*}, \\
& \bar{\varrho}^{+} \equiv-\frac{\hat{m}_{j}^{*}}{\bar{\rho}^{2}} \frac{\partial \hat{p}^{+}}{\partial x_{j}}+\frac{\hat{T}^{+}}{\bar{\rho}^{2}}\left(\bar{m}_{j} \frac{\partial \hat{T}^{*}}{\partial x_{j}}+\hat{m}_{j}^{*} \frac{\partial \bar{T}}{\partial x_{j}}\right)+\frac{\hat{Z}^{+}}{\bar{\rho}^{2}}\left(\bar{m}_{j} \frac{\partial \hat{Z}^{*}}{\partial x_{j}}+\hat{m}_{j}^{*} \frac{\partial \bar{Z}}{\partial x_{j}}\right) \ldots \\
& \cdots-\frac{\hat{T}^{+}}{S_{1} \operatorname{Re} \operatorname{Pr} \bar{\rho}^{2}} \frac{\partial^{2} \hat{T}^{*}}{\partial x_{j}^{2}}-\frac{\hat{Z}^{+}}{S_{1} \operatorname{Re} \operatorname{Pr} \bar{\rho}^{2}} \frac{\partial^{2} \hat{Z}^{*}}{\partial x_{j}^{2}}-\left(\frac{\bar{m}_{i} \hat{m}_{j}^{*}}{\bar{\rho}^{2}}+\frac{\hat{m}_{i}^{*} \bar{m}_{j}}{\bar{\rho}^{2}}\right) \frac{\partial \hat{m}_{i}^{+}}{\partial x_{j}} \cdots \\
& \cdots-\frac{1}{S_{1} \operatorname{Re}}\left(\frac{\hat{m}_{j}^{*}}{\bar{\rho}^{2}}\right)\left(\frac{\partial^{2} \hat{m}_{i}^{+}}{\partial x_{j}^{2}}+\frac{1}{3} \frac{\partial^{2} \hat{m}_{j}^{+}}{\partial x_{j} \partial x_{i}}\right)
\end{aligned}
$$

Based on previous studies on incompressible and compressible flows, $\overline{\mathbf{g}}^{+}$can be used to describe the sensitivity of the eigenvalue to arbitrary base flow modifications. Using the chain rule, we can express $\overline{\mathbf{g}}^{+}$in terms of primitive variables to derive the sensitivity of the eigenvalue to modifications of the base flow velocity and density profiles

$$
\begin{aligned}
\nabla_{\bar{u}_{i}} \lambda & =\bar{\rho} \bar{f}_{i}^{+} \\
\nabla_{\bar{\rho}} \lambda & =\bar{\varrho}^{+}-\frac{1}{\bar{\rho}^{2} \bar{K}_{2}} \bar{\psi}_{Z}^{+}-\frac{1}{\bar{\rho}^{2} \bar{K}_{1}} \bar{\psi}_{T}^{+}
\end{aligned}
$$

Once this has been calculated, the adjoint base flow equations can be solved.

To solve the adjoint base flow equations, we add time-derivative terms to the LHS of equations (9b)-(9d) and use a fourth-order Runge-Kutta scheme to march the axisymmetric form of the equations forward in time until the $l^{2}$-norm of the difference between the adjoint base flow state vectors at two succesive timsteps is less than $10^{-6}$. 

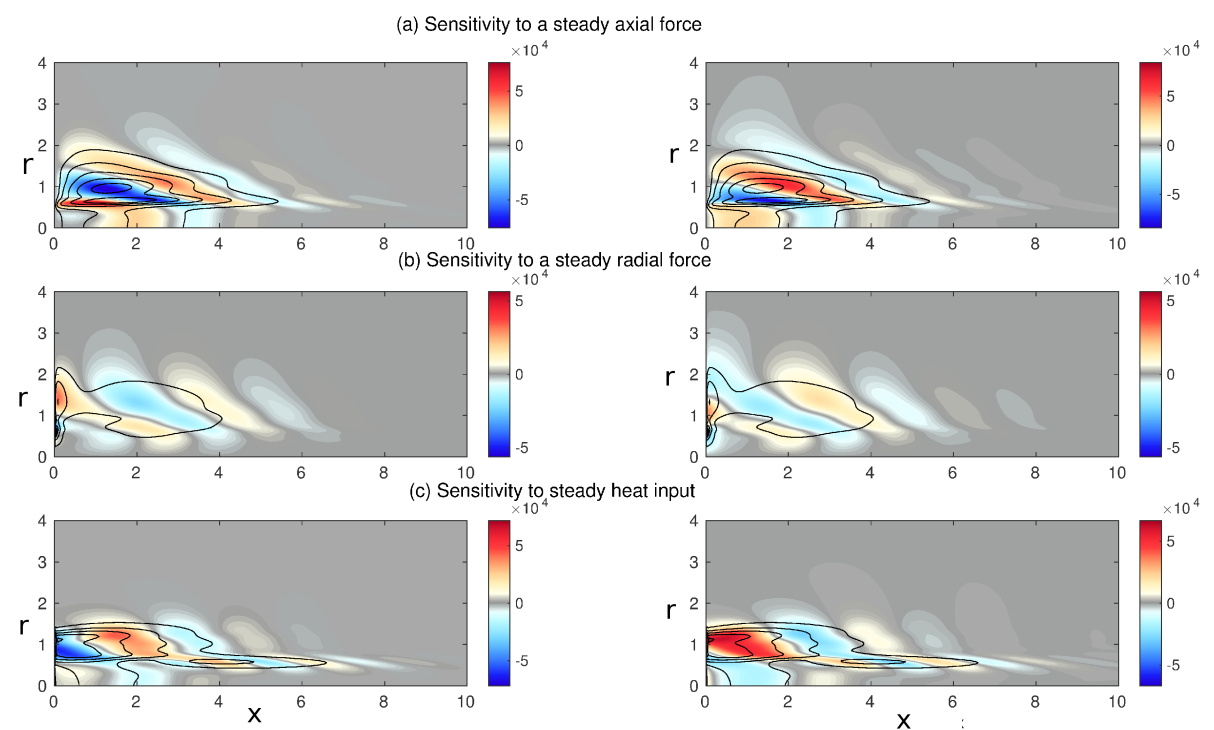

Figure 3: The sensitivity of the marginally unstable eigenvalue of a helium jet at $\operatorname{Re}=470$ to steady forcing, $\nabla_{\overline{\mathbf{F}}} \lambda$. The colours show the sensitivity of the growth rate, $\nabla \sigma$ (left), and frequency, $\nabla \omega$ (right) to a (a) steady axial body force, $\bar{F}_{x}$ (b) steady radial body force, $\bar{F}_{r}$, and (c) steady heat input, $\bar{\psi}_{T}$. The contour lines show the absolute value of $\nabla \lambda$ for each row. 
Figure 3 shows the adjoint base flow fields for the marginally unstable global mode of the isothermal helium jet at $R e=470$. The real part of the adjoint base flow fields determines the sensitivity of the growth rate to steady forcing, which determines whether the unstable mode is promoted or suppressed. The imaginary part determines the sensitivity of the frequency to steady forcing.

The sensitivities are oscillatory and the sensitivity patterns of the growth rate and frequency are out of phase - a peak in the sensitivity for the growth rate corresponds to a zero (a node) in the sensitivity for the frequency. Physically, this means that, at a particular location, a steady force can have maximal effect, either on the linear global mode growth rate, or on the linear global mode frequency, but not on both.

The adjoint base flow temperature describes the sensitivity to heat input. The flow is most sensitive to heat input just outside the helium jet, up to around 1.5 jet diameters from the injection plane. In this region, figure 3(c) (bottom left) shows that adding heat just outside the jet makes the flow more stable. Physically this is because heat addition reduces the density of the outer fluid. This agrees with the predictions of Srinivasan et al, [10] who used a local stability analysis to predict that heating in the ambient fluid near the jet nozzle can eliminate absolute instability. By comparing the bottom-left frame in figure 3(c) with the streamlines in figure 1(a), it is clear that the sensitivity contours approximately follow the streamlines. It appears therefore that the change induced by the heat source has most effect when it is advected onto the wavemaker region shown in figure 1(e). Consequently, the sensitivities in the regions outside the jet depend quite sensitively on the streamlines there and therefore on the degree of co-flow. The result that is most contrary to expectations is that heating the jet core around two diameters downstream has a stabilizing effect. In order to verify this, we carried out a check and found that heating the jet core two diameters downstream reduced the growth rate of the linear global mode, as predicted by the sensitivity analysis.

Unsurprisingly, radial momentum forcing has little influence, except in the shear layer at the jet exit plane (figure 3(b)). Axial momentum forcing has most 
influence just outside the jet, around the wavemaker region. In this region, figure 3 (a) shows that adding a force in the positive $x$-direction decreases the global mode frequency. The information in these figures is most instructive when it is combined in order to calculate the influence of a physical object, two of which we consider in the next section.

\section{Passive control using an axisymmetric control ring}

\subsection{The effect of an adiabatic control ring}

We now assume that the control force is provided by a thin ring at the same temperature as the fluid, which we call an adiabatic ring. The ring is at $\left(x_{c}, r_{c}\right)$, centred on the jet axis, and provides a force on the flow that is equal and opposite to the drag force on the ring. In this linear stability analysis, the steady base flow causes a steady drag force, and the growth of perturbations causes an unsteady drag force. The ring is thin, so the non-dimensional steady and unsteady components of the drag force can be modelled by those on a cylinder:

$$
\begin{aligned}
\overline{\mathbf{F}}(x, r) & =-\alpha \bar{\rho}|\overline{\mathbf{u}}| \overline{\mathbf{u}} \delta^{2}\left(x-x_{c}, r-r_{c}\right), \\
\mathbf{f}^{\prime}(x, r, t) & =\hat{\mathbf{f}}(x, r) e^{\lambda t} \\
\text { where } \hat{\mathbf{f}}(x, r) & =-\left(\alpha \hat{\rho}|\overline{\mathbf{u}}| \overline{\mathbf{u}}+\alpha \bar{\rho}|\overline{\mathbf{u}}| \hat{\mathbf{u}}+\alpha \bar{\rho} \frac{\overline{\mathbf{u}} \cdot \hat{\mathbf{u}}}{|\overline{\mathbf{u}}|} \overline{\mathbf{u}}\right) \delta^{2}\left(x-x_{c}, r-r_{c}\right) .
\end{aligned}
$$

The coefficient $\alpha$ equals $d_{w} C_{D}$, where $C_{D}$ is the drag coefficient and $d_{w}$ is the wire diameter non-dimensionalized by the jet diameter. We set $C_{D}=1.5$, based on numerical drag calculations.[26] We set $d_{w}=0.1$, which corresponds to a maximum local Reynolds number around 50, because this is below the Reynolds number at which the ring causes its own self-excited oscillations.[26] Therefore, the linearized drag force oscillates only at the frequency of the global mode, $\lambda$.

The changes in the eigenvalue due to the steady and unsteady components of the drag force are $\delta \lambda_{\overline{\mathbf{F}}}=\left\langle\overline{\mathbf{m}}^{+}, \overline{\mathbf{F}}\right\rangle$ and $\delta \lambda_{\mathbf{f}^{\prime}}=\left\langle\hat{\mathbf{m}}^{+}, \hat{\mathbf{f}}\right\rangle$. These are summed to obtain the total change in the eigenvalue, $\delta \lambda_{\text {drag }}$. Figure 4 shows the change in 

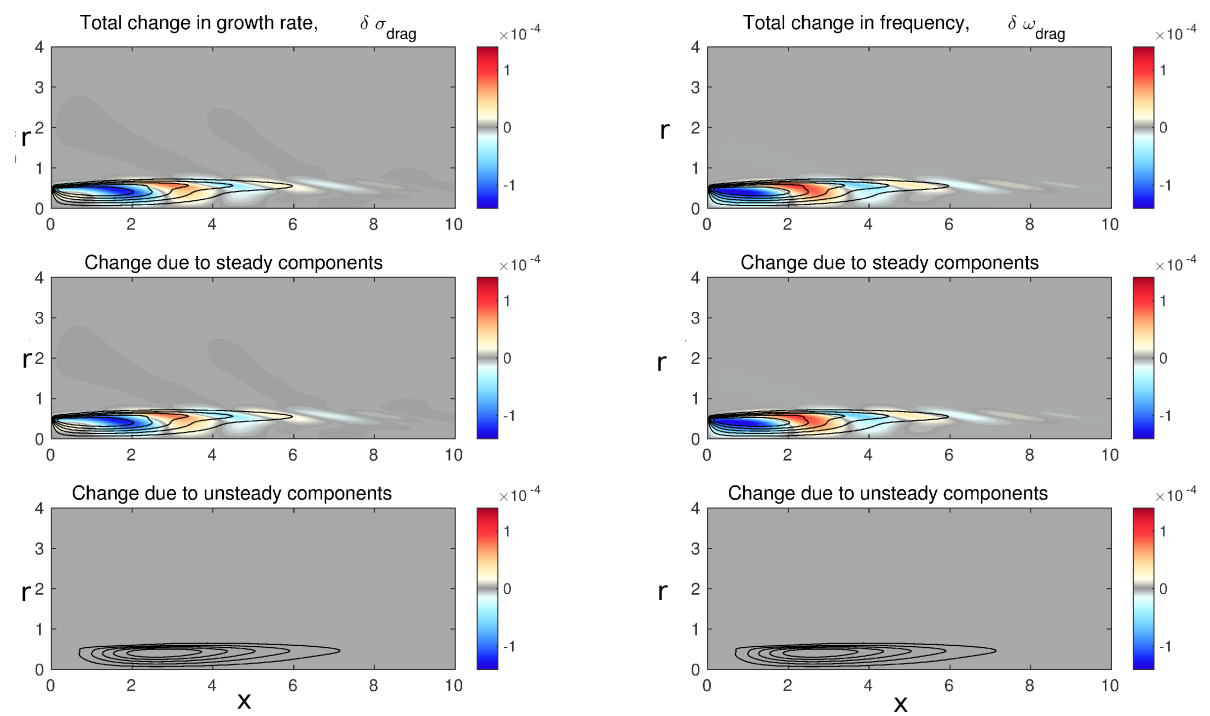

Figure 4: The predicted change (scaled by $C_{D} d_{w}$ ) in the marginally unstable eigenvalue of a helium jet due to the drag on a thin axisymmetric control ring, $\delta \lambda_{\text {drag }}$. The colours show the real (left) and imaginary (right) parts of the total change in the eigenvalue, $\delta \lambda_{\text {drag }}$ (top), the change due to the steady component of the drag force, $\delta \lambda_{\overline{\mathbf{F}}}$ (middle), and the change due to the unsteady component of the drag force, $\delta \lambda_{\mathbf{f}^{\prime}}$ (bottom). The contours show the absolute value of $\delta \lambda$ for each row. The shading on all the plots is equal. 
the growth rate and frequency (scaled by $C_{D} d_{w}$ ) as a function of the location of the control ring. This shows where the control ring has most influence. The drag from the ring increases as the flow velocity at the ring increases, so the ring has little effect where the base flow is slow, even if the eigenmode is quite sensitive to momentum forcing there. This is why figure 4 (sensitivity to a control ring) has highest amplitudes in the jet region $(0<r<0.5)$ despite the fact that figure 3 (sensitivity to steady forcing) has highest amplitudes outside the jet region.

The ring has maximum influence when placed at $\left(x_{c}, r_{c}\right)=(1.0,0.43)$, at which point it is stabilizing. Furthermore, it decreases the oscillation frequency when placed at $0<x<2.0$ and increases the frequency when placed at $2.0<$ $x<3.2$. It is interesting to note that the steady component of the drag force influences the eigenvalue around 3 times more than the unsteady component.

\subsection{The effect of a heated control ring}

We now consider the additional influence of heat transfer from a hot ring. Chandler [27] calculated the steady and unsteady components of the heat transfer to be:

$$
\begin{aligned}
\bar{\psi}_{T}(x, r) & =c_{\psi} d_{w}^{\eta}|\overline{\mathbf{m}}|^{\eta}\left(T_{w}-\bar{T}\right) \delta^{2}\left(x-x_{c}, r-r_{c}\right), \\
\psi_{T}^{\prime}(x, r, t) & =\hat{\psi}_{T}(x, r) e^{\lambda t} \\
\text { where } \quad \hat{\psi}_{T}(x, r) & =c_{\psi} d_{w}^{\eta}|\overline{\mathbf{m}}|^{\eta}\left(\left(T_{w}-\bar{T}\right) \eta \frac{\mathbf{m}^{\prime} \cdot \overline{\mathbf{m}}}{|\overline{\mathbf{m}}|^{2}}-T^{\prime}\right) \delta^{2}\left(x-x_{c}, r-r_{c}\right) .
\end{aligned}
$$

$T_{w}$ is the non-dimensional wire temperature and $c_{\psi}$ and $\eta$, which are functions of the Nusselt and Reynolds numbers, are taken to be $c_{\psi}=58.3$ and $\eta=0.33$. [27] We consider a small increase in the non-dimensional ring temperature, $T_{w}=$ 0.01. At an ambient temperature of $300 \mathrm{~K}$, this corresponds to a dimensional increase of 3 degrees. The changes in the eigenvalue due to the steady and unsteady components of the heat transfer are $\delta \lambda_{\bar{T}}=\left\langle\bar{T}^{+}, \bar{\psi}_{T}\right\rangle$ and $\delta \lambda_{T^{\prime}}=$ $\left\langle\hat{T}^{+}, \hat{\psi}_{T}\right\rangle$. These are summed with the changes due to the drag $\left(\delta \lambda_{\text {drag }}\right)$ to 

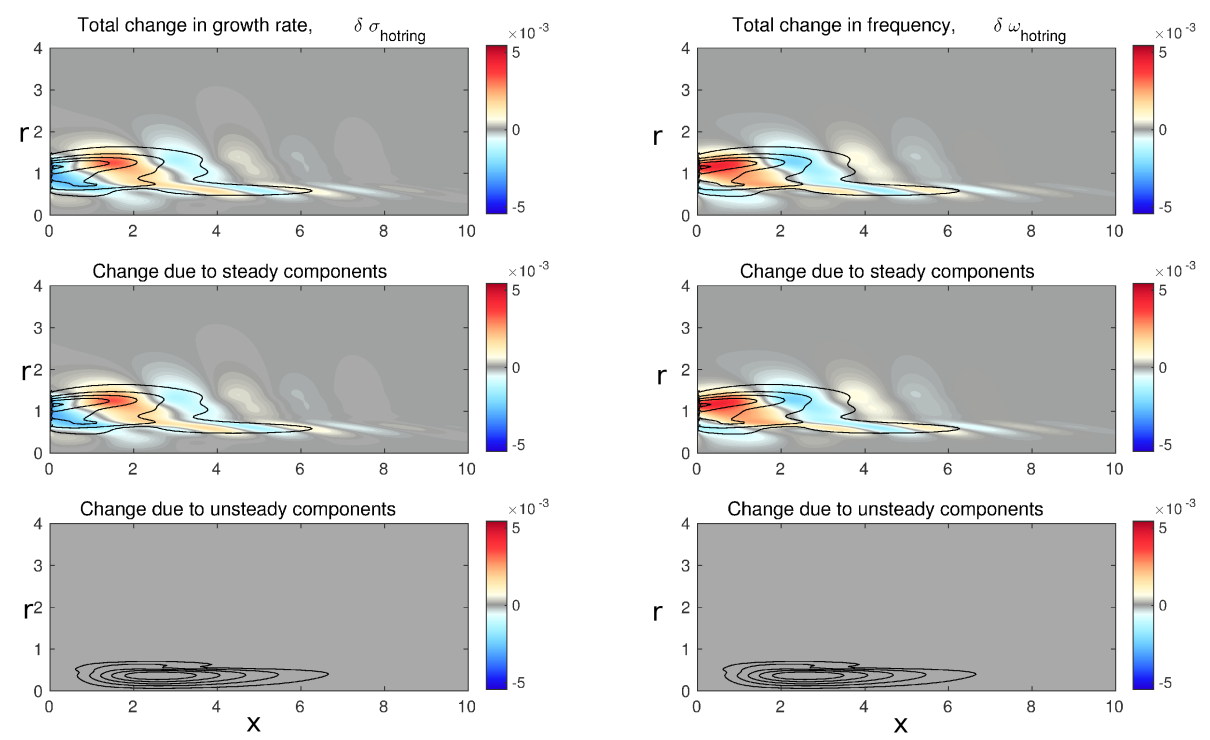

Figure 5: The predicted change in the marginally unstable eigenvalue of a helium jet, $\delta \lambda_{\text {hotring }}$, due to the drag and heat transfer from a thin hot ring with $d_{w}=0.1$ and $T_{w}=0.01$. The colours show the real (left) and imaginary (right) parts of the total change in the eigenvalue, $\delta \lambda_{\text {hotring }}$ (top), the change due to the steady components of the drag and heat transfer, $\delta \lambda_{\overline{\mathbf{F}}}+\delta \lambda_{\bar{T}}$ (middle), and the change due to the unsteady components of the drag and heat transfer, $\delta \lambda_{\mathbf{f}^{\prime}}+\delta \lambda_{T^{\prime}}$ (bottom). The contours show the absolute value of $\delta \lambda$ for each row. The shading on all the plots is equal.

obtain the total change in the eigenvalue, $\delta \lambda_{\text {hotring }}$. Figure 5 shows these total changes as a function of the location of the hot ring. When it is hot, the wire has more influence in the low speed regions outside the jet than when it is adiabatic, as shown by the fact that figure 5 is more similar to figure $3(\mathrm{c})$ than figure 4 is to figure $3(\mathrm{a})$. This is because the heat transfer depends much less on the local velocity than the drag force does (the exponent of velocity is $\eta=0.33$ in equation (13a) but 2 in equation (12a)). The hot ring stabilizes the flow when placed just outside the shear layer, around $0.0<x<2.0$, because it reduces the density there. It also has a strong effect when placed at a larger radius. This is because the density reduction caused by the heat transfer is advected along the streamlines to the jet. This advection depends strongly on the streamlines and therefore on the degree of co-flow. 


\section{Conclusions}

In this paper, we have performed a structural sensitivity analysis and a base flow sensitivity analysis of the stability of a low-density jet. We have used direct numerical simulations of the direct and adjoint low Mach number Navier-Stokes equations: in nonlinear form to find the steady base flow, and in linear form to simulate the evolution of infinitesimal perturbations. With an Arnoldi algorithm, we have calculated the direct and adjoint global modes for the structural sensitivity analysis and have combined this with a Lagrangian approach for the base flow sensitivity analysis. We have produced maps of the regions of the flow that are most sensitive to localized open loop steady forcing. This forcing can take the form of a body force, a mass source, and a heat source.

We have found that the maximum of the structural sensitivity, which is sometimes known as the wavemaker region, lies in the shear layer 2.66 jet diameters downstream of the exit plane. This flow is locally absolutely unstable from the exit plane to 5 jet diameters downstream and has maximum absolute growth rate at the exit plane. Our global analysis shows that the most sensitive location for open loop steady forcing is the area around the shear layer, around 2 jet diameters downstream of the exit plane. This forcing can change the growth rate and frequency of the primary global mode. We find that the influence of steady forcing and heat injection is advected by the flow outside the jet. This means that these results depend on the streamlines around the jet, which are sensitive to the degree of co-flow around the jet.

We have used these maps to calculate the influence of a ring placed in the flow. When the ring is at the same temperature as the flow, it influences the flow only through its drag. In this case, the influence of the steady component of the drag force far outweighs the influence of the unsteady component. Depending on its axial position, the ring changes the growth rate or frequency of the primary global mode, in a manner that should be possible to measure experimentally. This result depends very little on the streamlines outside the jet because the velocity is small there, so there is little drag. When the ring is hotter than the 
flow, it also influences the flow through heat input. Again, the influence of the steady component far outweighs the influence of the unsteady component. The hot ring has most influence when placed in the outer edge of the shear layer. It is also influential when placed outside the jet because the expanded gas is advected towards the jet by the surrounding flow. In the slow-moving outer flow, heat transfer from the ring is more influential than drag from the ring because heat transfer depends less strongly on the local velocity than the drag does. Again, this should be possible to measure experimentally, although when the ring is placed outside the jet, its predicted influence depends significantly on the streamlines, which may be difficult to replicate in an experiment.

\section{Appendix A. Grid convergence checks}

In this appendix, we calculate the base flow and linear global stability on several different meshes in order to assess the reliability and convergence of the results. Table A.1 compares the growth rate and frequency of the least stable direct and adjoint global mode, while varying the number of grid points, the domain size, and the time-step used in the matrix-free time-stepping algorithm. Meshes M1 and M3 have roughly the same spatial resolution and can be labelled as low-resolution cases. Meshes M2 and M4 have roughly the same spatial resolution and can be labelled as mid-resolution cases. Meshes M5 and M5a have the same spatial resolution and can be labelled as high-resolution cases. The difference between the mid- and high-resolution cases is small enough for us to be confident that these results are well-converged.

In the absence of trucation errors, the adjoint eigenvalues would be the complex conjugate of the direct eigenvalues. In this study, however, the discretization errors between the direct and adjoint algorithms are not the same because a continuous-adjoint scheme is used. The adjoint eigenvalue must, therefore, be checked to ensure that it is correct. The discrepancy between the direct and adjoint eigenvalues for the least stable mode is shown in table A.1. The discrepancy between the two is never more than $0.25 \%$ and gets smaller with 


\begin{tabular}{|c|c|c|c|c|c|c|c|c|c|c|}
\hline & $X_{\max }$ & $R_{\max }$ & $S x$ & $S r$ & $\Delta t$ & $\sigma_{d i r}$ & $S t_{d i r}$ & $\sigma_{a d j}$ & $S t_{a d j}^{*}$ & $\begin{array}{c}\text { Relative } \\
\text { discrepancy } \\
(\%)\end{array}$ \\
\hline M1 & 36.0 & 4.0 & 1025 & 127 & 0.005 & 0.0126 & 0.1623 & 0.0111 & 0.1620 & 0.24 \\
\hline M2 & 24.0 & 4.0 & 1025 & 181 & 0.005 & 0.0009 & 0.1646 & 0.0013 & 0.1647 & 0.07 \\
\hline M3 & 36.0 & 6.0 & 1025 & 181 & 0.005 & 0.0124 & 0.1628 & 0.0110 & 0.1626 & 0.18 \\
\hline M4 & 36.0 & 4.0 & 1449 & 181 & 0.005 & -0.0011 & 0.1650 & -0.0003 & 0.1646 & 0.25 \\
\hline M5 & 36.0 & 4.0 & 2049 & 255 & 0.005 & -0.0048 & 0.1655 & -0.0058 & 0.1656 & 0.11 \\
\hline M5a & 36.0 & 4.0 & 2049 & 255 & 0.001 & -0.0030 & 0.1658 & -0.0033 & 0.1658 & 0.03 \\
\hline
\end{tabular}

Table A.1: Domain size ( $X_{\max }, R_{\max }$ ), number of grid points ( $S x, S r$ ), and time-step $(\Delta t)$ used in the simulations to validate the global stability analysis. The growth rate $\sigma$, and frequency (Strouhal number $S t$ ) of the least stable direct and adjoint global modes are shown.

increasing grid-resolution and decreasing time-step. This is in agreement with the first-order temporal accuracy of the time-stepping scheme [19]. We use mesh M5 in this study.

\section{Appendix B. Validation of sensitivity maps}

In this section, we validate our sensitivity maps and check whether the predicted changes in the eigenvalue are the same as those found by numerical simulation.

First, we validate the sensitivity to arbitrary base flow modifications. We perturb each component of the base flow by a small amount $\delta \overline{\mathbf{q}}=\varepsilon \overline{\mathbf{q}}$ separately and calculate the perturbed eigenvalue using our direct eigenvalue solver. We compare the normalized change in the eigenvalue $\delta \lambda_{a c t}=[\lambda(\overline{\mathbf{q}}+\varepsilon \overline{\mathbf{q}})-\lambda(\overline{\mathbf{q}})] / \varepsilon$ to the predicted change in the eigenvalue $\delta \lambda_{\text {pred }}=\left\langle\overline{\mathbf{g}}^{+}, \delta \overline{\mathbf{q}}\right\rangle$. The results are shown in Table B.2 and show good agreement between the predicted and actual eigenvalues. Note that for $\delta \bar{Z}$, we need to use the chain rule to take into account the change in the density field caused by the change in mixture fraction, $\delta \lambda_{\text {pred }}=$ $\left\langle\bar{\psi}_{Z}{ }^{+}-\bar{K}_{2} \bar{\rho}^{2}, \delta \bar{Z}\right\rangle$.

Next, we validate the sensitivity to steady forcing. We consider the effect of 


\begin{tabular}{c|c|c|c} 
& $\delta \bar{m}_{x}$ & $\delta \bar{m}_{r}$ & $\delta \bar{Z}$ \\
\hline \hline Predicted & $0.0185+1.0451 \mathrm{i}$ & $0.0116+0.0218 \mathrm{i}$ & $0.1255+0.5085 \mathrm{i}$ \\
Actual & $0.0180+1.0452 \mathrm{i}$ & $0.0114+0.0219 \mathrm{i}$ & $0.1270+0.5051 \mathrm{i}$ \\
Relative discrepancy (\%) & 0.05 & 0.70 & 0.72
\end{tabular}

Table B.2: Comparison between the predicted and actual change in the eigenvalue due to an arbitrary base flow modification of the form $\delta \overline{\mathbf{q}}=\varepsilon \overline{\mathbf{q}}$.

\begin{tabular}{c|c|c|c} 
Steady force & $\delta \lambda_{\text {pred,SF }}$ & $\delta \lambda_{\text {pred,BFM }}$ & $\delta \lambda_{\text {act }}$ \\
\hline \hline $\bar{f}_{x}=-0.01 e^{-100\left((x-1.0)^{2}+(r-0.5)^{2}\right)}$ & $-0.0193-0.0054 \mathrm{i}$ & $-0.0197-0.0052 \mathrm{i}$ & $-0.0203-0.0054 \mathrm{i}$
\end{tabular}

Table B.3: Comparison between the predicted and actual change in the eigenvalue due to a steady force. We compare the actual change in the eigenvalue $\left(\delta \lambda_{a c t}\right)$ with the change predicted using the sensitivity to steady forcing $\left(\delta \lambda_{\text {pred,SF }}\right)$ and the change predicted using the sensitivity to base flow modifications with the forced base flow $\left(\delta \lambda_{\text {pred,BFM }}\right)$

a momentum source term described by $\bar{f}_{x}=-0.01 e^{-100\left((x-1.0)^{2}+(r-0.5)^{2}\right)}$. The change in the eigenvalue due to this steady forcing can be calculated in three ways. Firstly, we predict the change in the eigenvalue using the sensitivity to steady forcing framework, $\delta \lambda_{\text {pred,SF }}=\left\langle\bar{m}_{x}^{+}, \delta \bar{f}_{x}\right\rangle$. Secondly, we add the forcing term to the RHS of the NS equations and obtain a new steady base flow, $\bar{q}+\delta \bar{q}$. The change in the eigenvalue can then be predicted using the sensitivity to base flow modifications, $\delta \lambda_{\text {pred,BFM }}=\left\langle\overline{\mathbf{g}}^{+}, \delta \bar{q}\right\rangle$. Thirdly, the new eigenvalue can be calculated using the direct eigenvalue solver on the new steady base flow. In the linear approximation, all three methods should give the same answer. The results are shown in Table B.3. The predicted change in the eigenvalue using the sensitivity to steady forcing and base flow modifications agree well with the actual change in the eigenvalue calculated using the direct eigenvalue solver. The small discrepancy is due to the use of the continuous-adjoint and firstorder accuracy of the time-stepping scheme used to calculate the eigenvalues. 

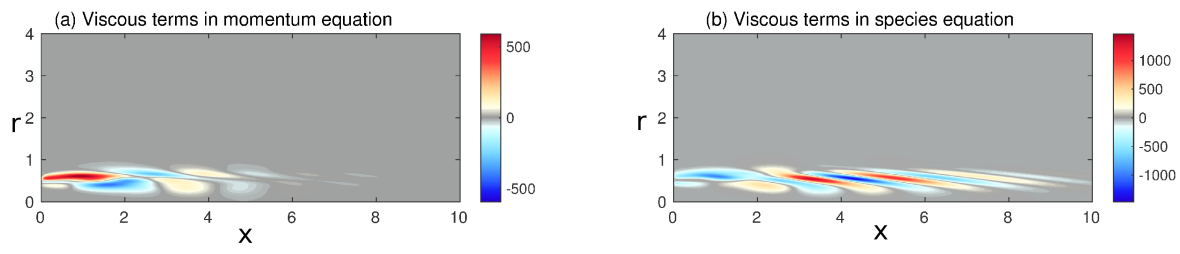

Figure C.6: The sensitivity of the marginally unstable eigenvalue of a helium jet at $\operatorname{Re}=470$ to viscous effects. The colours show the sensitivity of the growth rate to viscous terms in (a) the momentum equation (1b) $\nabla_{v i s c, m_{i}} \lambda$, and (b) the species conservation equation (1c) $\nabla_{\text {visc }, Z} \lambda$. The magnitudes can be compared directly to those in figure 3 .

\section{Appendix C. Analysis of assumptions}

In this study, we have made a number of simplifying assumptions to understand the fundamental mechanisms that are involved in the control of global instability in a low-density jet. The first assumption is that of uniform transport properties. In order to confirm the validity of this assumption for the flow configuration that we have studied, we calculate the sensitivity of the eigenvalue to the viscous terms on the RHS of equations (1b-1c), given by

$$
\begin{gathered}
\nabla_{v i s c, m_{i}} \lambda=\frac{\bar{m}_{i}^{+}}{S_{1} \operatorname{Re}}\left(\frac{\partial^{2} \bar{u}_{i}}{\partial x_{j}^{2}}+\frac{1}{3} \frac{\partial^{2} \bar{u}_{i}}{\partial x_{j} x_{i}}\right)+\frac{\hat{m}_{i}^{+}}{S_{1} \operatorname{Re}}\left(\frac{\partial^{2} \hat{u}_{i}^{*}}{\partial x_{j}^{2}}+\frac{1}{3} \frac{\partial^{2} \hat{u}_{i}^{*}}{\partial x_{j} x_{i}}\right), \\
\nabla_{v i s c, Z} \lambda=\frac{\bar{Z}^{+}}{S_{1} \operatorname{Re} S c} \frac{\partial^{2} \bar{Z}}{\partial x_{j}^{2}}+\frac{1}{S_{1} \operatorname{Re} S c}\left(\frac{\hat{Z}^{+}}{\bar{\rho}}+\bar{K}_{2} \hat{p}^{+}\right) \frac{\partial^{2} \hat{Z}^{*}}{\partial x_{j}^{2}} .
\end{gathered}
$$

These quantify the importance of viscous effects in determining the linear growth rate and frequency of the global mode. The sensitivity maps are shown in figure C.6. We notice that the growth rate is more sensitive to the viscous terms in the species equation than the viscous terms in the momentum equation. The sensitivity is, however, at least one order of magnitude smaller than the sensitivity to steady forcing, shown in figure 3 . We conclude, therefore, that for the flow configuration in this study, viscous effects do not have a large effect on the linear global stability analysis. For flows at lower Reynolds number, or those dominated by buoyancy effects, we expect that viscous effects will be more influential. 

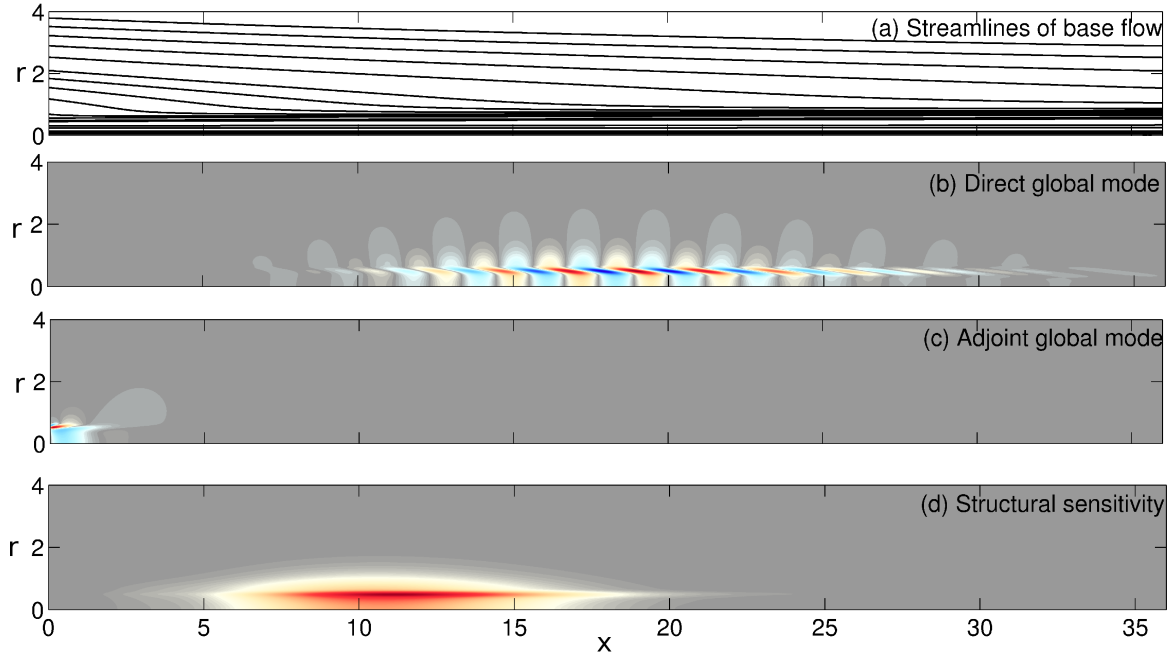

Figure C.7: (a) Streamlines of the base flow of an isothermal jet with $S_{1}=4.0$ at $\operatorname{Re}=2000$. (b) The direct global mode and (c) the adjoint global mode, shown as the real part of the axial momentum. (d) The structural sensitivity, with a maximum of $3 \times 10^{5}$.

Our second main assumption concerns the boundary conditions used in the simulation. The flow configuration that we have studied corresponds to a jet exiting from a hole into a large open space. Upstream of the jet exit plane, density variations would be negligible and the flow would be linearly stable. At the jet exit plane, however, the flow is absolutely unstable. In this study, the jet exit plane corresponds to the inlet to the computational domain, and we have imposed homogeneous Dirichlet boundary conditions on all perturbations there, similar to what has been done in previous studies [3, 18]. The numerical tools used here did not allow us to model the flow upstream of the jet exit plane, or to set other boundary conditions at the jet exit plane. Changing the boundary condition at the inlet will have an effect on the global stability analysis. We expect that the growth rate and frequency will change, and that the sensitivity maps will shift upstream. Qualitatively, however, we expect that the sensitivity maps will still exhibit the same features as those in this study.

Finally, it is worth discussing how the results of this study apply to flows at higher Reynolds numbers. We carried out a global stability analysis for a 
marginally unstable low-density jet with $S_{1}=4.0$ at $R e=2000$. The results are shown in figure C.7 and are qualitatively similar to those in figure 1. The main difference is that the convective non-normality is stronger at higher Reynolds numbers. This is indicated by the greater spatial separation between the maxima of the direct and adjoint global modes. The strength of the non-normality is also indicated by the maximum of the structural sensitivity. At $\operatorname{Re}=2000$, the maximum of the structural sensitivity is almost two orders of magnitude greater than at $\operatorname{Re}=470$. This means that the growth rate and frequency of the global mode are much more sensitive to small changes in the feedback mechanism driving the global mode, and also to small changes in the base flow. Any small change to the flow such as that induced by a hot-wire can have a large effect on the flow dynamics.

[1] K. R. Sreenivasan, S. Raghu, D. Kyle, Absolute instability in variable density round jets, Experiments in Fluids 7 (1989) 309-317.

[2] P. A. Monkewitz, D. W. Bechert, B. Barsikow, B. Lehmann, Self-excited oscillations and mixing in a heated round jet, Journal of Fluid Mechanics 213 (1990) 611-639.

[3] L. Lesshafft, P. Huerre, P. Sagaut, M. Terracol, Nonlinear global modes in hot jets, Journal of Fluid Mechanics 554 (2006) 393-409.

[4] W. Coenen, A. Sevilla, A. L. Snchez, Absolute instability of light jets emerging from circular injector tubes, Physics of Fluids 20 (7).

[5] W. Coenen, A. Sevilla, The structure of the absolutely unstable regions in the near field of low-density jets, Journal of Fluid Mechanics 713 (2012) $123-149$.

[6] W. Coenen, L. Lesshafft, X. Garnaud, A. Sevilla, Global instability of lowdensity jets, Journal of Fluid Mechanics 820 (2017) 187-207.

[7] J.-M. Chomaz, P. Huerre, L. G. Redekopp, A frequency selection criterion 
in spatially developing flows, Studies in Applied Mathematics 84 (2) (1991) $119-144$.

[8] P. A. Monkewitz, P. Huerre, J.-M. Chomaz, Global linear stability analysis of weakly non-parallel shear flows, Journal of Fluid Mechanics 251 (1993) $1-20$.

[9] M. P. Hallberg, P. J. Strykowski, Open-loop control of fully nonlinear selfexcited oscillations, Physics of Fluids 20 (041703).

[10] V. Srinivasan, M. P. Hallberg, P. J. Strykowski, Viscous linear stability of axisymmetric low-density jets: Parameters influencing absolute instability, Physics of Fluids 22 (024103).

[11] M. P. Hallberg, V. Srinivasan, P. Gorse, P. J. Strykowski, Suppression of global modes in low-density axisymmetric jets using coflow, Physics of Fluids 19 (014102).

[12] O. Marquet, D. Sipp, L. Jacquin, Sensitivity analysis and passive control of cylinder flow, Journal of Fluid Mechanics 615 (2008) 221-252. doi:10.1017/S0022112008003662.

[13] D. C. Hill, A theoretical approach for analyzing the re-stabilization of wakes, AIAA Paper 92-0067.

[14] P. A. McMurtry, W.-H. Jou, J. J. Riley, R. W. Metcalfe, Direct numerical simulations of a reacting mixing layer with chemical heat release, AIAA Journal 24 (1986) 962-970.

[15] M. P. Hallberg, P. J. Strykowski, On the universality of global modes in low-density axisymmetric jets, Journal of Fluid Mechanics 569 (2006) 493507.

[16] L. K. B. L. Li, M. P. Juniper, Lock-in and quasiperiodicity in a forced hydrodynamically self-excited jet, Journal of Fluid Mechanics 726 (2013) 624-655. 
[17] R. Chakravarthy, L. Lesshafft, P. Huerre, Global stability of buoyant jets and plumes, Journal of Fluid Mechanics 835 (2018) 654-673.

[18] J. W. Nichols, P. J. Schmid, J. J. Riley, Self-sustained oscillations in variable-density round jets, Journal of Fluid Mechanics 582 (2007) 341376. doi:10.1017/S0022112007005903.

[19] G. J. Chandler, M. P. Juniper, J. W. Nichols, P. J. Schmid, Adjoint algorithms for the Navier-Stokes equations in the low Mach number limit, Journal of Computational Physics 231 (4) (2012) 1900-1916.

[20] A. Michalke, Survey on jet instability theory, Progress in Aerospace Sciences 21 (1984) 159-199.

[21] L. Lesshafft, O. Marquet, Optimal velocity and density profiles for the onset of absolute instability in jets, Journal of Fluid Mechanics 662 (2010) 398-408.

[22] E. Akervik, L. Brandt, D. S. Henningson, J. Hoepffner, O. Marxen, P. Schlatter, Steady solutions of the Navier-Stokes equations by selective frequency damping, Physics of Fluids 18 (068102). doi:10.1063/1.2211705.

[23] X. Garnaud, L. Lesshafft, P. J. Schmid, P. Huerre, Modal and transient dynamics of jet flows, Physics of Fluids 25 (044103).

[24] F. Giannetti, P. Luchini, Structural sensitivity of the first instability of the cylinder wake, Journal of Fluid Mechanics 581 (2007) 167-197. doi:10.1017/S0022112007005654.

[25] O. Marquet, D. Sipp, L. Jacquin, J.-M. Chomaz, Multiple timescale and sensitivity analysis for the passive control of the cylinder flow, 5th AIAA Theoretical Fluid Mechanics Conference, 23-26 June 2008, Seattle, Washington (2008).

[26] G. J. Sheard, K. Hourigan, M. C. Thompson, Computations of the drag coefficients for low-Reynolds-number flow past rings, Journal of Fluid Mechanics 526 (2005) 257-275. 
[27] G. J. Chandler, Sensitivity analysis of low-density jets and flames, Ph.D. thesis, University of Cambridge (2010). 